\title{
Device Modeling and Numerical Characterization of Perovskite/Si, Perovskite/CIGS and all-Perovskite Tandem Solar Cells
}

\author{
Saddam Hussain ${ }^{1}$, Adnan Daud Khan ${ }^{2}$ \\ 1 1,2 US.Pakistan center for advanced studies in energy (USPCAS-E), University of engineering \\ and technology (UET) Peshawar, Pakistan. \\ saddamahmad313@gmail.com ${ }^{1}$ \\ Received: 16 December, Revised: 26 December, Accepted: 27 December
}

\begin{abstract}
From the past couple of years, the high-power conversion efficiency (PCE) of $>25 \%$ and low-cost fabrication of single-junction perovskite photovoltaic cells have gained great attention from researchers. The bandgap tunability of these solar cells makes them an attractive and ideal candidate for tandem solar cell applications. The PCEs above than the single-junction solar cells theoretical Shockley-Queisser (SQ) radiative efficiency limit (31\%-33\%) can be achieved by harvesting a wide fraction of solar spectrum using multijunction solar cells. In perovskite tandem (double-junction) solar cells, a wide-bandgap perovskite top cell is combined with either narrow-bandgap bottom cells of dissimilar materials like silicon ( $\mathrm{Si}$ ) and copper indium gallium selenide (CIGS) or with low bandgap perovskite solar cell. In this work, we have simulated perovskite/Si (PVK/Si), perovskite/CIGS (PVK/CIGS) and perovskite/perovskite (PVK/PVK) tandem solar cells and estimated 28.73\%, 20.31\% and 26.06\% PCEs. The highest conversion efficiency is shown by PVK/Si tandem cells among others because of the suitable bandgap for tandem applications. Our work will guide the researchers for obtaining ultra-high conversion efficiency solar cells.
\end{abstract}

Keywords - Tandem solar cells, SunSolve, optical modeling, efficiencies, evaluation.

\section{INTRODUCTION}

The metal-halide perovskite solar cells have gained the attention of researchers in the past couple of years and became a hot topic of the photovoltaics (PV) community because at low manufacturing costs these solar cells have the potential to achieve a high PCEs [1][2][3]. The high performance of these solar cells is because of its remarkable optoelectronic properties like higher carrier mobility, longer diffusion lengths, high absorption and light to electrical conversion. Due to intense research efforts in the past few years from researchers in the photovoltaic community across the world have helped the conversion efficiencies of single-junction (SJ) PSCs to climb from $<14 \%$ in 2013 to $25.2 \%$ in 2019[4]. The singlejunction PSCs conversion efficiencies are approaching the Shockley-Queisser (SQ) theoretical limits (31-33\%)[5]. In order to harvest the maximum fraction of the solar spectrum and go beyond the single junction $\mathrm{S}-\mathrm{Q}$ limit, the multi-junction PSCs should be investigated. Due to this reason, the researchers are intended towards solar cells having double and triple junctions, which can achieve PCEs of greater than $42 \%$ under standard solar irradiance[6]. The multijunction tandem design aims to reduce energy losses due to the thermalization of light excited carriers by combining a stack of different bandgap semiconductor absorbers. The high energy photons are absorbed by the top subcell (wide-bandgap) of the stack and the bottom subcell (low bandgap) absorbs the low energy photons. In this way, the solar spectrum's wide portion is harvested. In the past 3-4 years, all the tandem cells have gained rapid progress due to the developments in low and high bandgap perovskite solar cells. In late 2014, for the first time, the PCE of $13.4 \%$ and $17 \%$ respectively reported by McGehee and Ballif groups separately for 4-T MAPI3/Si [7] and MAPbI3/multi-crystalline $\mathrm{Si}$ [8] tandem solar cells. More recently, the Ho-Baillie group combined (FAPbI3)0.83(MAPbBr3)0.17 with homojunction c-Si textured on the rear side in a monolithic architecture and showed a PCE of $21.8 \%$ having cell area of $16 \mathrm{~cm} 2$ [9]. Their work provides a path that leads to the commercialization of low-cost tandem solar cells. Perovskites are ideal partners of CIGS solar cells for tandem applications. Recently, for perovskite/CIGS tandems a PCE of 24.6\% [10] and 21.6\%[11] for 4-T and 2-T is achieved by researchers. All-perovskite based tandem cells are also fabricated in both 4-T and 2-T architecture. Recently, a PCE of 23\% [12] and 24.8\%[13] is reported for all-perovskite 4-T and 2-T tandems respectively. In our work, we used the SunSolve simulation tool from PV Lighthouse [14] for the optical simulation of monolithic 2-T PVK/c-Si, PVK/CIGS, and PVK/PVK tandem solar cells and estimated and compared their efficiencies. Our simulations are based on measured complex refractive indices ( $\mathrm{n}$ and $\mathrm{k}$ ) also known as optical constants for the different layers of the design stack. With our proposed device architectures, we have studied losses (reflection and parasitic absorption in each layer) and gains in absorber layers and have estimated a PCEs of $28.73 \%, 20.31 \%$ and $26.06 \%$ for PVK/c-Si, PVK/CIGS and PVK/PVK tandem solar cells under AM 1.5G 1000W m-2 and 300K temperature. In our simulations the PVK/c-Si tandem architecture exhibited 
greater efficiency compared to PVK/CIGS and PVK/PVK tandem architectures.

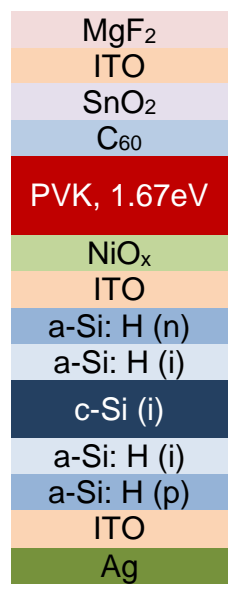

(a)

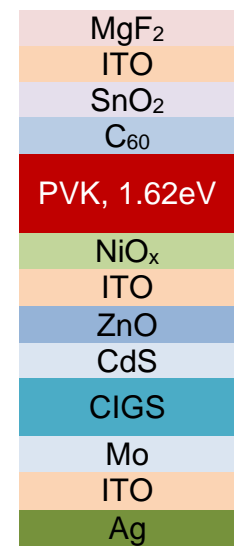

(b)

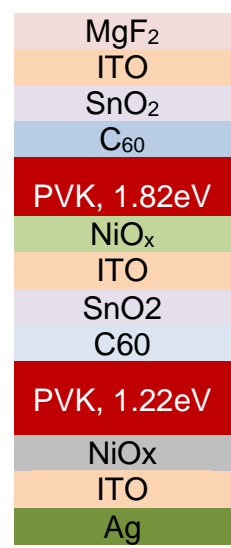

(c)
Figure 1: The device structure of monolithic 2-T PVK/c-Si (a), PVK/CIGS and (b) and PVK/PVK tandem solar cells (c) used in simulations.

\section{DEVICE MODELING AND SIMULATIONS}

The device architectures for the various tandem solar cells in our simulations are shown in figure 1 . In the $\mathrm{PVK} / \mathrm{c}-\mathrm{Si}$ tandem architecture, the top subcell absorber bandgap is $1.67 \mathrm{eV}$ and the bottom subcell absorber ( $\mathrm{Si}$ ) bandgap is $1.1 \mathrm{eV}$. Here the $\mathrm{MgF} 2$ is used as an anti-reflection layer to reduce reflection losses and improve the overall performance of the solar cell. The values of refractive indices ( $n$ and $k$ ) for each layer in the stack are obtained from the Refractive Index Library of SunSolve. The top and bottom subcells are serially interconnected using the recombination layer of indium tin oxide (ITO). In the PVK/CIGS architecture, the top subcell absorber bandgap is $1.62 \mathrm{eV}$ and the bottom subcell absorber (CIGS) bandgap is $1.15 \mathrm{eV}$. The data for complex refractive indices of CIGS is obtained from the literature [15] and the remaining layers of the stack are obtained from the Refractive Index Library of SunSolve. The top and bottom subcells of the PVK/CIGS are interconnected by ITO as a recombination layer. Similarly, for PVK/PVK device stack, the front and rear absorbers used have a bandgap of $1.82 \mathrm{eV}$ and $1.22 \mathrm{eV}$. The complex refractive indices data for the absorber layers is obtained from [16] and the remaining layers of the stack are obtained from the Refractive Index Library of SunSolve. The subcells are interconnected using ITO as a recombination layer (RL). Simulation software is known as SunSolve which is introduced by PV Lighthouse [14] for the solar cell optical simulations. The simulation method of SunSolve is based on the combination of Monte Carlo ray tracing (MRT) and thinfilm optics which determines the optical losses and gains in various layers of solar cells for a chosen spectrum of light. For a user-defined spectrum, the magnitude of photon flux is calculated and integrated over the wavelength to determine the photon current density. The photo-generated current $J_{G}$ in the stack of solar cells equates to the photo-current which can be pulled out with the help of electrodes. In simulations using
SunSolve, we have used the perfect Lambertian reflector $(\Lambda=1)$ scattering model at the bottom of the solar cell stack.

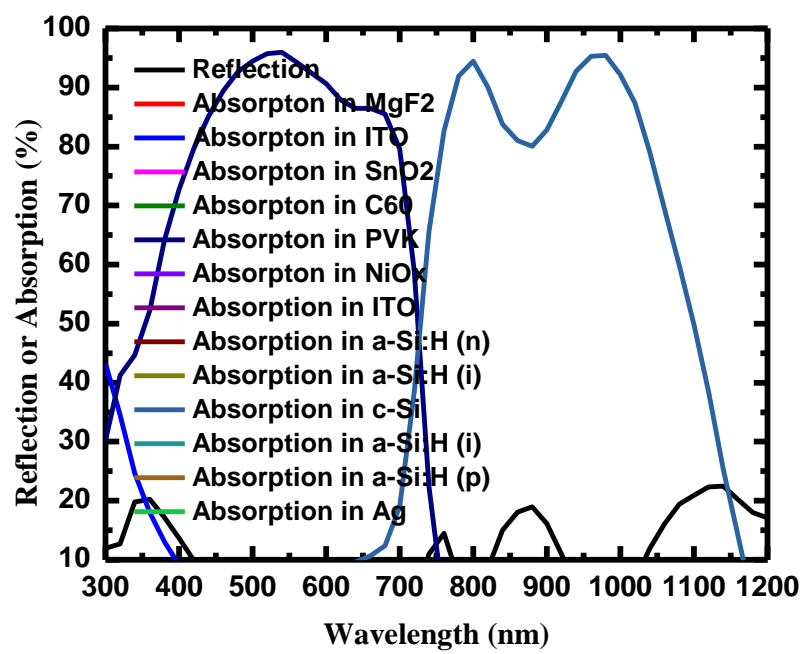

Figure 2: Reflection and absorption losses simulated in the device stack of $\mathrm{PVK} / \mathrm{c}-\mathrm{Si}$ tandem cell.

\section{RESULTS AND DISCUSSION}

There are three different tandem architectures are simulated and compared in this research work. These are a) PVK/c-Si, b) PVK/CIGS and c) PVK/PVK tandem solar cells. Losses due to reflection and parasitic absorption in non-absorber layers are calculated and plotted. The quantum efficiencies for the proposed models are plotted whose detail is given below.

\section{Perovskite-Silicon Tandem Solar Cell}

Without substantial change in manufacturing costs, the output power of crystalline silicon solar panels can be increased by combining with wide bandgap PSCs. A $\sim 1.1 \mathrm{eV}$ c$\mathrm{Si}$ combined with wide bandgap perovskites of $1.6-1.75 \mathrm{eV}$ is an ideal tandem combination to achieve a PCE of more than $30 \%$ [17]. Here we have combined $1.67 \mathrm{eV}$ top subcell perovskite absorber with c-Si bottom subcell as shown in figure 1(a). All the layers of the stack are optimized using layer by layer optimization technique whose detail is listed in table 1 and a $20.4 \mathrm{~mA} \mathrm{~cm}-2$ matched current density is obtained. The losses taking place in the device stack due to reflection from the front surface and parasitic absorption in the non-active layers which do not contribute to the electron-hole generation are plotted in figure 2 for the solar spectrum from $300 \mathrm{~nm}$ to $1200 \mathrm{~nm}$. In the solar cell stack, about $7.87 \%(3.65 \mathrm{~mA} \mathrm{~cm}-2)$ of the incoming radiation is reflected from the front surface.

The perovskite/c-Si tandem stack photons to electrons conversion are higher than the individual subcells of the tandem stack. Because the solar spectrum large portion is harvested due to a combination of active layers of different bandgaps. The EQE plot in figure 3 shows that the photons of higher energy are absorbed by top subcells while the photons of lower energy i.e. greater wavelengths are absorbed by the bottom subcell of the tandem stack. The refinements made here in conjunction with the best-reported fill factor $(\mathrm{FF}=79 \%)$ and open-circuit voltage $\left(\mathrm{V}_{\mathrm{oc}}=1.771 \mathrm{~V}\right)$ and as reported in [18] along with our calculated matched current density, we estimate a PCE of $28.73 \%$. 
Table 1: Materials used in the optical simulation using SunSolve, their thicknesses range for optimization and optimized thicknesses of PVK/c-Si tandem solar cell. (MAPI= Methylammonium lead iodide)

\begin{tabular}{|c|c|c|c|}
\hline Cell & Layers & $\begin{array}{c}\text { Thickness } \\
\text { range }(\mathrm{nm})\end{array}$ & $\begin{array}{c}\text { Optimized } \\
\text { thickness } \\
(\mathbf{n m})\end{array}$ \\
\hline \multirow{6}{*}{$\begin{array}{c}\text { Perovskite } \\
\text { top subcell } \\
(1.67 \mathrm{eV})\end{array}$} & MgF2 & $50-250$ & 100 \\
\hline & ITO & $60-200$ & 62 \\
\hline & $\mathrm{SnO}_{2}$ & $5-50$ & 15 \\
\hline & $\mathrm{C}_{60}$ & $5-50$ & 10 \\
\hline & MAPI & $100-1500$ & 330 \\
\hline & $\mathrm{NiO}_{\mathbf{x}}$ & $10-100$ & 20 \\
\hline RL & ITO & $10-300$ & 20 \\
\hline \multirow{7}{*}{$\begin{array}{c}\text { Silicon } \\
\text { bottom } \\
\text { subcell } \\
(1.22 \mathrm{eV})\end{array}$} & a-Si:H (n) & 10 & 10 \\
\hline & a-Si:H (i) & 5 & 5 \\
\hline & c-Si & $1-1000 \mu \mathrm{m}$ & $130 \mu \mathrm{m}$ \\
\hline & a-Si:H (i) & 5 & 5 \\
\hline & a-Si:H (p) & 10 & 10 \\
\hline & ITO & 150 & 150 \\
\hline & Ag & 300 & 300 \\
\hline
\end{tabular}

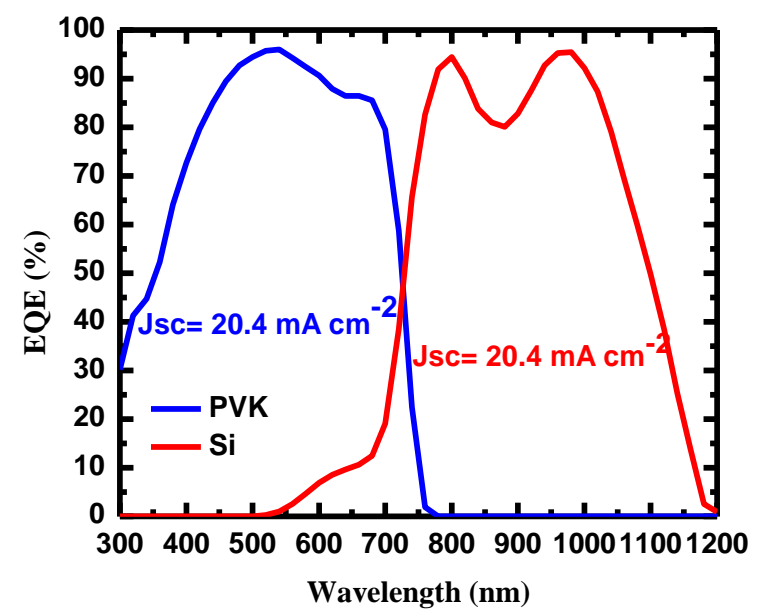

Figure 3: EQE of tandem PVK/c-Si solar cell stack for the solar spectrum of $300 \mathrm{~nm}$ to $1200 \mathrm{~nm}$. Here the high energy photons are absorbed in the top subcell (blue line) and the low energy photons are absorbed by the bottom subcell (red line).

\section{Perovskite-CIGS Tandem Solar Cell}

In the tandem PVK/CIGS solar cell simulations, a perovskite solar cell having an absorber bandgap of $1.62 \mathrm{eV}$ is employed as a top subcell and CIGS solar cell with absorber bandgap $=1.15 \mathrm{eV}$ is employed as bottom subcell as shown in figure 1(b). The detail of various layer thicknesses and their optimized values are listed in table 2. The tandem stack is optimized using layer by layer optimization technique and a $19.4 \mathrm{~mA} \mathrm{~cm}^{-2}$ matched current density is achieved. In figure 4 the breakdown of losses taking place in the tandem stack is plotted for a spectrum range of $300 \mathrm{~nm}$ to $1200 \mathrm{~nm}$, which shows both reflection and parasitic absorption taking place in the proposed model of perovskite/CIGS tandem solar cell. Here about $6.30 \%(2.92 \mathrm{~mA} \mathrm{~cm}$ ) of the incoming solar spectrum $\left(46.3 \mathrm{~mA} \mathrm{~cm}^{-2}\right)$ is reflected back from the front surface of the
Table 2: Materials used in the optical simulation of SunSolve, their thicknesses range for optimization and optimized thicknesses of perovskite/cSi tandem solar cell. (MAPI= Methylammonium lead iodide)

\begin{tabular}{|c|c|c|c|}
\hline Cell & Layers & Thickness range (nm) & $\begin{array}{c}\text { Optimized } \\
\text { thickness } \\
(\mathbf{n m})\end{array}$ \\
\hline \multirow{6}{*}{$\begin{array}{c}\text { Perovskite } \\
\text { top subcell } \\
(1.62 \mathrm{eV})\end{array}$} & MgF2 & $50-250$ & 100 \\
\hline & ITO & $60-200$ & 62 \\
\hline & $\mathrm{SnO}_{2}$ & $5-50$ & 15 \\
\hline & $\mathrm{C}_{60}$ & $5-50$ & 10 \\
\hline & MAPI & $100-1500$ & 330 \\
\hline & $\mathrm{NiO}_{x}$ & $10-100$ & 20 \\
\hline $\mathbf{R L}$ & ITO & $10-300$ & 20 \\
\hline \multirow{6}{*}{$\begin{array}{c}\text { CIGS } \\
\text { bottom } \\
\text { subcell } \\
(1.15 \mathrm{eV})\end{array}$} & $\mathrm{ZnO}$ & 10 & 10 \\
\hline & Cds & 5 & 5 \\
\hline & CIGS & $1-1000 \mu \mathrm{m}$ & $1.05 \mu \mathrm{m}$ \\
\hline & Mo & 10 & 10 \\
\hline & ITO & 150 & 150 \\
\hline & Ag & 300 & 300 \\
\hline
\end{tabular}

device stack which is lower from reflection losses taking place in tandem $\mathrm{PVK} / \mathrm{c}-\mathrm{Si}$ stack $(7.87 \%)$. This loss is reasonably reduced by the use of $\mathrm{MgF}_{2}$ as an anti-reflection layer. Large parasitic losses are taking place in front of the transporting layers of the stack.

The tandem cell overall performance is limited by the less efficient subcell of the stack. The EQE of the perovskite/CIGS stack is the summation of its subcells EQEs. In figure 5, EQE of the perovskite/CIGS tandem stack is shown for the solar spectrum range from $300 \mathrm{~nm}$ to $1200 \mathrm{~nm}$ (absorbing region). The high bandgap top subcell harvest maximin photons near $550 \mathrm{~nm}$ wavelength which is a high energy fraction of the incoming solar spectrum. The narrow bandgap $(1.15 \mathrm{eV})$ bottom subcell (CIGS) has high photons to electrons ratio near $850 \mathrm{~nm}$ wavelength which is an intermediate energy fraction of incoming solar spectrum. By using these modifications and guidelines in conjunction with best-reported Voc $=1.49 \mathrm{~V}$, and $\mathrm{FF}=70.2 \%$ by [11] with our calculated $\mathrm{J}_{\mathrm{sc}}$ of $19.5 \mathrm{~mA} \mathrm{~cm} \mathrm{~cm}^{-2}$ for tandem PVK/CIGS solar cell stack, we estimate a PCE of $20.31 \%$. 


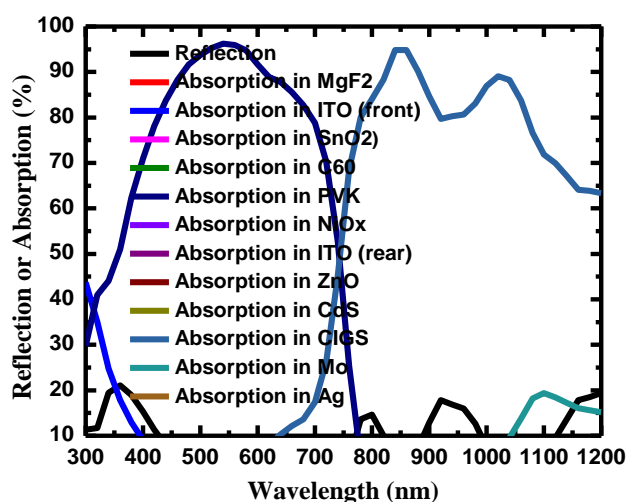

Figure 4: Reflection and absorption losses simulated in the device stack of PVK/CIGS tandem solar cell.

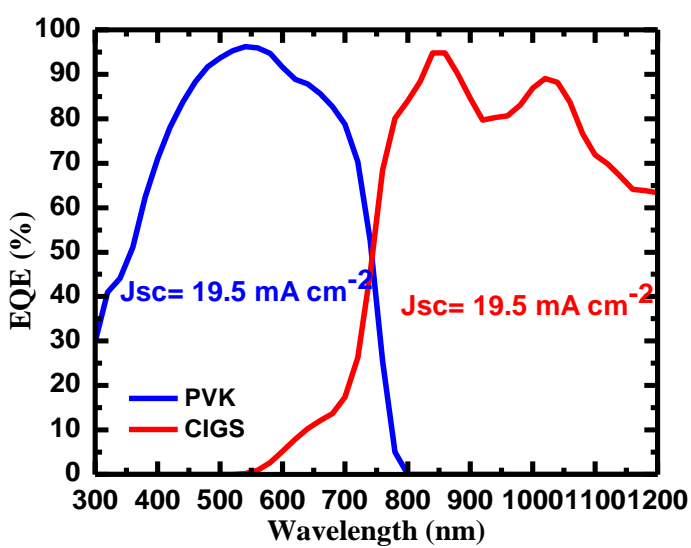

Figure 5: EQE of 2-T monolithic tandem PVK/CIGS solar cell is plotted. Here the high energy photons are absorbed by the top subcell (blue line) and the low energy photons are absorbed by the bottom subcell (red line).

\section{All-Perovskite Tandem Solar Cells}

The architecture used in the simulations of tandem PVK/PVK solar cells is given in figure 1(c). For the tandem architecture of PVK/PVK solar cells, a cell having high-Eg from $\sim 1.7$ to $1.9 \mathrm{eV}$ is used as a top subcell and a cell having low bandgap from $\sim 1.1$ to $1.3 \mathrm{eV}$ is used as bottom subcell [19]. The top subcell absorbs those photons whose energy is greater than its bandgap, while the bottom subcell absorbs those whose energy is greater from its bandgap and less than the top subcell bandgap. The final performance of the two-terminal tandem stack is greatly dependent on the matching of current between the subcells. The total current of the tandem cell is limited by a less efficient subcell of the stack. The low bandgap bottom subcell is relatively thicker and absorbs the infrared light passing from a wide bandgap top subcell. In our simulation, a perovskite cell with a wide-bandgap $(1.82 \mathrm{eV})$ absorber layer is employed as a top subcell and a perovskite with a narrow bandgap (1.22eV) absorber is employed as rear subcell in the PVK/PVK tandem stack. The subcells are interconnected through ITO which acts as a recombination layer. The thicknesses of both active layers in the tandem stack are varied until the current matching is achieved. A matched current density of $18 \mathrm{~mA} \mathrm{~cm} \mathrm{~cm}^{-2}$ among the tandem stack subcells is achieved at thicknesses of $330 \mathrm{~nm}$ and $1250 \mathrm{~nm}$ for front and rear subcells respectively. The thickness range of each layer and optimized thickness values are listed in table 3 . The breakdown of losses in the all-perovskite tandem stack for a solar spectrum range from $300 \mathrm{~nm}$ to $1200 \mathrm{~nm}$ is plotted in figure 6, which shows the reflection and parasitic absorptions along with absorption in active layers. The higher parasitic absorption occurs in $\mathrm{MgF}_{2}$, front ITO and top subcell fullerene $\left(\mathrm{C}_{60}\right)$, which is $0.627,0.456$, and, $0.410 \mathrm{~mA} \mathrm{~cm}{ }^{-2}$. Nearly $6.17 \%$ corresponds to $2.86 \mathrm{~mA} \mathrm{~cm}^{-2}$ of the incoming radiation is reflected from the front of the tandem cell. In the design, $10.6 \%\left(4.89 \mathrm{~mA} \mathrm{~cm}^{-2}\right)$ of the incident radiation is escaped from the front of the solar cell. The recombination layer has very fewer absorption losses, which shows its significance to be used as a recombination layer. The all-perovskite tandem stack photons to electrons conversion are higher than the individual subcells of the tandem stack. Because the solar spectrum large portion is harvested due to a combination of active layers of different bandgaps.

Table 3: Materials used in the optical simulation of SunSolve, their thicknesses range for optimization and optimized thicknesses of perovskite/perovskite tandem solar cell.

\begin{tabular}{|c|c|c|c|}
\hline Cell & Layers & $\begin{array}{l}\text { Thickness } \\
\text { range (nm) }\end{array}$ & $\begin{array}{c}\text { Optimized } \\
\text { thickness }(\mathrm{nm})\end{array}$ \\
\hline \multirow{6}{*}{$\begin{array}{c}\text { Perovskite } \\
\text { top subcell } \\
(1.82 \mathrm{eV})\end{array}$} & $\mathrm{MgF} 2$ & $50-250$ & 100 \\
\hline & ITO & $60-200$ & 62 \\
\hline & $\mathrm{SnO}_{2}$ & $5-50$ & 15 \\
\hline & $\mathrm{C}_{60}$ & $5-50$ & 10 \\
\hline & $\mathrm{MA}_{0.9} \mathrm{Cs} 0.1 \mathrm{~Pb}\left(\mathrm{I}_{0.6} \mathrm{Br}_{0.4}\right)_{3}$ & $100-1500$ & 330 \\
\hline & $\mathrm{NiO}_{\mathrm{x}}$ & $10-100$ & 20 \\
\hline $\mathbf{R L}$ & ITO & $10-300$ & 20 \\
\hline \multirow{6}{*}{$\begin{array}{c}\text { Perovskite } \\
\text { bottom } \\
\text { subcell } \\
(1.22 \mathrm{eV})\end{array}$} & $\mathrm{SnO}_{2}$ & $5-50$ & 15 \\
\hline & $\mathrm{C}_{60}$ & $5-50$ & 10 \\
\hline & $\mathrm{CH}_{3} \mathrm{NH}_{3} \mathrm{~Pb}_{0.5} \mathrm{Sn}_{0.5} \mathrm{I}_{3}$ & $100-1500$ & 1250 \\
\hline & $\mathrm{NiO}_{\mathrm{x}}$ & $10-100$ & 20 \\
\hline & ITO & 150 & 150 \\
\hline & $\mathrm{Ag}$ & 300 & 300 \\
\hline
\end{tabular}

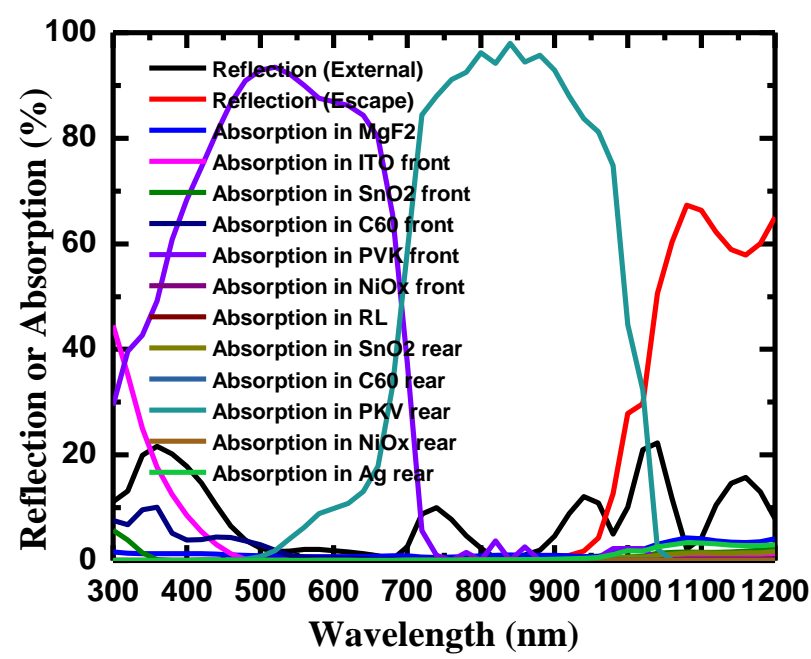

Figure 6: Reflection and absorption losses simulated in the device stack of perovskite/perovskite tandem solar cell. 


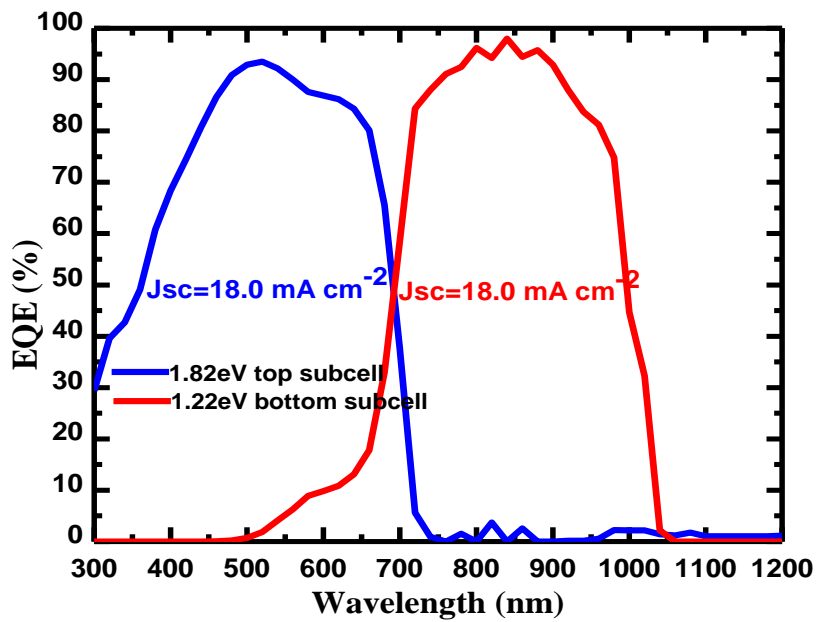

Figure 7: EQE of PVK/PVK tandem solar cell is plotted. Here the high energy photons are absorbed by the top subcell (blue line) and the low energy photons are absorbed by the bottom subcell (red line).

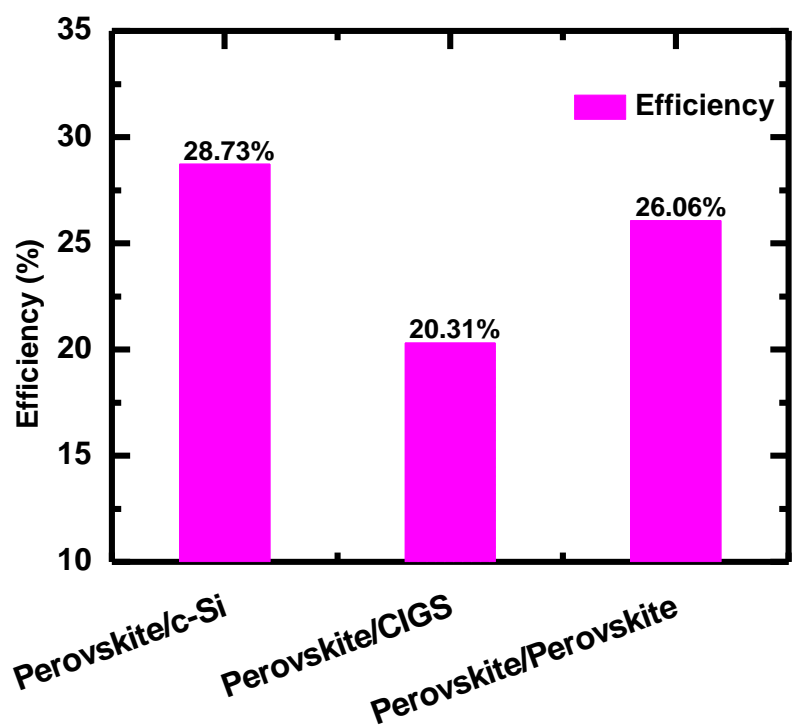

Figure 8: Comparison of three different tandem configurations that are simulated in our work.

The EQE plot in figure 7 shows that the photons of higher energy are absorbed by top subcell while the bottom subcell of the tandem stack absorbs photons of lower energy i.e. greater wavelengths. The fringes in the EQE plot of the rear subcell are due to interferences which leads to limit the light-generated current of the rear subcell and can be reduced by thinning the non-perovskite layers to 5nm [20]. By using these design guidelines in conjunction with $\mathrm{Voc}=1.98 \mathrm{~V}$ and $\mathrm{FF}=73 \%$ from best-reported tandem PVK/PVK solar cell [16], we estimated a $26.06 \%$ PCE. Here the efficiency of tandem PVK/CIGS solar cell is inferior to the $\mathrm{PVK} / \mathrm{c}-\mathrm{Si}$ and tandem $\mathrm{PVK} / \mathrm{PVK}$ solar cell as shown in figure 8.

\section{CONCLUSION}

In conclusion to this work, we optically simulated three types of 2-T monolithic tandem solar cells which are PVK/c-Si, PVK/CIGS, and PVK/PVK solar cells. For the simulation of tandem PVK/c-Si solar cell, we used a $\sim 1.1 \mathrm{eV}$ c-Si bottom subcell and a $1.67 \mathrm{eV}$ perovskite top subcell and achieved a $20.4 \mathrm{~mA} \mathrm{~cm}-2$ current density after optimization using layer by layer optimization and estimated a PCE of $28.73 \%$. Then we optically simulated a 2-T monolithic tandem PVK/CIGS solar cell and determined various losses in the stack. A $19.4 \mathrm{~mA} \mathrm{~cm}-$ 2 matched current density is achieved at $695 \mathrm{~nm}$ and $1050 \mathrm{~nm}$ optimized thicknesses of top and bottom absorber layers respectively and PCE of $20.31 \%$ is estimated. For modeling of the tandem PVK/PVK solar cell, a wide-bandgap $(1.82 \mathrm{eV})$ subcell on top of a narrow bandgap (1.22eV) subcell is employed to make a tandem PVK/PVK solar cell. An $18.0 \mathrm{~mA}$ cm-2 matched current density is calculated and a PCE of $26.06 \%$ is estimated. By reduction of the non-radiative recombination and optical losses in perovskite-based solar cells can lead the multijunction perovskite solar cells performances beyond the numbers we calculated here in our simulations.

\section{ACKNOWLEDGMENT}

I would like to acknowledge the support from USPCAS-E UET Peshawar, Arizona State University (ASU) and USAID during my research work.

\section{REFERENCES}

[1] J.-P. Correa-Baena et al., "Promises and challenges of perovskite solar cells," Science (80-. )., vol. 358, no. 6364, pp. 739-744, 2017.

[2] T. Leijtens, K. A. Bush, R. Prasanna, and M. D. McGehee, "Opportunities and challenges for tandem solar cells using metal halide perovskite semiconductors," Nat. Energy, vol. 3, no. 10, p. 828, 2018.

[3] G. E. Eperon, M. T. Hörantner, and H. J. Snaith, "Metal halide perovskite tandem and multiple-junction photovoltaics," Nat. Rev. Chem., vol. 1, no. 12, p. 95, 2017.

[4] "Best Research-Cell Efficiencies, available online. [Accessed: 20 October 2019], 2019.”

[5] W. Shockley and H. J. Queisser, "Detailed balance limit of efficiency of p-n junction solar cells," J. Appl. Phys., vol. 32, no. 3, pp. 510519, 1961.

[6] A. De Vos, "Detailed balance limit of the efficiency of tandem solar cells,” J. Phys. D. Appl. Phys., vol. 13, no. 5, p. 839, 1980.

[7] P. Löper et al., "Organic-inorganic halide perovskite/crystalline silicon four-terminal tandem solar cells," Phys. Chem. Chem. Phys., vol. 17, no. 3, pp. 1619-1629, 2015.

[8] C. D. Bailie et al., "Semi-transparent perovskite solar cells for tandems with silicon and CIGS," Energy Environ. Sci., vol. 8, no. 3, pp. 956963, 2015.

[9] J. Zheng et al., " $21.8 \%$ efficient monolithic perovskite/homo-junctionsilicon tandem solar cell on $16 \mathrm{~cm} 2$," ACS Energy Lett., vol. 3, no. 9, pp. 2299-2300, 2018.

[10] "Imec Perovskite/CIGS tandem cell with Record Efficiency of 24.6 percent Paves the Way for Flexible Solar Cells and High-Efficiency Building-Integrated PV 2018.[Online]. Available:(https://www.imecint.com/en/articles/perovskite-cigs-tandem- cell-with-rec," 2018.

[11] M. Jost et al., "21.6\%-Efficient Monolithic Perovskite/Cu (In, Ga) Se2 Tandem Solar Cells with Thin Conformal Hole Transport Layers for Integration on Rough Bottom Cell Surfaces," ACS Energy Lett., vol. 4, no. 2, pp. 583-590, 2019.

[12] D. Zhao et al., "Four-terminal all-perovskite tandem solar cells achieving power conversion efficiencies exceeding 23\%," ACS Energy Lett., vol. 3, no. 2, pp. 305-306, 2018.

[13] S. Albrecht et al., "Monolithic perovskite/silicon-heterojunction tandem solar cells processed at low temperature," Energy Environ. Sci., vol. 9, no. 1, pp. 81-88, 2016.

[14] ""PVlighthouse,." [Online]. https:/www.pvlighthouse.com.au/sunsolve.".
Available: 
[15] P. D. Paulson, R. W. Birkmire, and W. N. Shafarman, "Optical characterization of CuIn 1- $\mathrm{x}$ Ga $\mathrm{x}$ Se 2 alloy thin films by spectroscopic ellipsometry," J. Appl. Phys., vol. 94, no. 2, pp. 879-888, 2003.

[16] A. Rajagopal et al., "Highly efficient perovskite-perovskite tandem solar cells reaching $80 \%$ of the theoretical limit in photovoltage," Adv. Mater., vol. 29, no. 34, p. 1702140, 2017.

[17] [17] Z. Song, C. Chen, C. Li, R. A. Awni, D. Zhao, and Y. Yan, "Widebandgap, low-bandgap, and tandem perovskite solar cells," Semicond. Sci. Technol., vol. 34, no. 9, p. 93001, 2019.

[18] J. Y. Zhengshan and Z. C. Holman, "Predicting the Efficiency of the Silicon Bottom Cell in a Two-Terminal Tandem Solar Cell," in 2017 IEEE 44th Photovoltaic Specialist Conference (PVSC), 2017, pp. 32503253.

[19] D. Zhao et al., "Efficient two-terminal all-perovskite tandem solar cells enabled by high-quality low-bandgap absorber layers," Nat. Energy, vol. 3 , no. 12, p. $1093,2018$.

[20] M. T. Hörantner et al., "The potential of multijunction perovskite solar cells,” ACS Energy Lett., vol. 2, no. 10, pp. 2506-2513, 2017. 